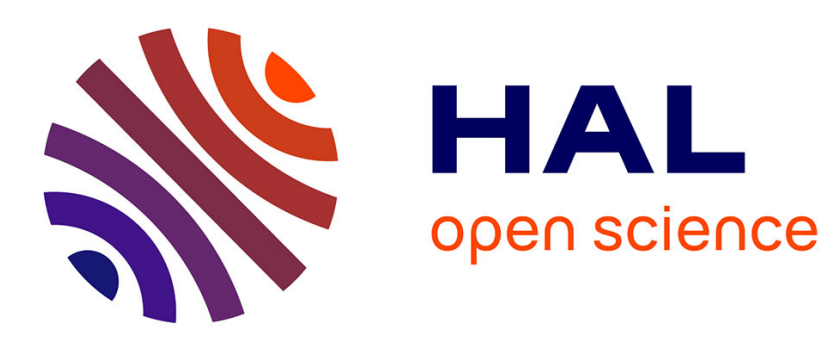

\title{
Two-field surface pattern control via marginally stable magnetorheological elastomers
}

\author{
Erato Psarra, Laurence Bodelot, Kostas Danas
}

\section{To cite this version:}

Erato Psarra, Laurence Bodelot, Kostas Danas. Two-field surface pattern control via marginally stable magnetorheological elastomers. Soft Matter, 2017, 13 (37), pp.6576 - 6584. 10.1039/C7SM00996H . hal-01627525

\section{HAL Id: hal-01627525 \\ https://hal-polytechnique.archives-ouvertes.fr/hal-01627525}

Submitted on 21 Dec 2017

HAL is a multi-disciplinary open access archive for the deposit and dissemination of scientific research documents, whether they are published or not. The documents may come from teaching and research institutions in France or abroad, or from public or private research centers.
L'archive ouverte pluridisciplinaire HAL, est destinée au dépôt et à la diffusion de documents scientifiques de niveau recherche, publiés ou non, émanant des établissements d'enseignement et de recherche français ou étrangers, des laboratoires publics ou privés. 


\title{
Two-field surface pattern control via marginally stable magnetorheo- logical elastomers $\dagger$
}

\author{
Erato Psarra, ${ }^{a}$ Laurence Bodelot, ${ }^{a}$ and Kostas Danas ${ }^{a, *}$
}

Received 18th May 2017, Accepted 24th August 2017

Soft Matter

DOI: $10.1039 / \mathrm{c} 7 \mathrm{sm00996h}$

The stability and post-bifurcation of a non-linear magnetoelastic film/substrate block is experimentally exploited to obtain active control of surface roughness. The non-intuitive interplay between magnetic field and elastic deformation owes to material and geometry selection, namely a ferromagnetic particle composite film bonded on a compliant passive foundation. Cooperation of two otherwise independent loading mechanisms-mechanical pre-compression and magnetic field-allows to bring the structure near a marginally stable state and then destabilize it with either magnetic or mechanical fields. We demonstrate for the first time that the critical magnetic field is a decreasing function of pre-compression and vice versa. The experimental results are then probed successfully with full-field finite element simulations at large strains and magnetic fields. The magnetoelastic coupling allows for the reversible on/off control of surface wrinkling under adjustable critical magnetic and mechanical fields, thus this study constitutes a first step towards realistic active haptic and morphing devices.

\section{Introduction}

Mechanically stiff films or layers resting on passive compliant substrates are known to undergo mechanical instabilities under uniaxial compressive loads. The principal solution of such material systems is unique and stable until the load exceeds a critical value, which causes the film to bifurcate into a geometric mode influenced by the (visco-)elastic nature of the substrate. ${ }^{1-4}$ The resulting critical buckling mode is determined by the stiffness contrast, or in simple cases, the substrate-to-film shear moduli ratio $G_{s} / G_{f}$, as well as the geometrical constraints of the stucture. ${ }^{4-7}$ In the post-bifurcation regime, periodic wrinkling is observed within a $G_{s} / G_{f}$ range that is lower than a threshold $\sim 0.5 .^{8-10}$ Upon further compression, wrinkles evolve into more complex morphologies (e.g., folds, creases, ridges). ${ }^{4,5,10-13}$ Mechanically induced instabilities in planar geometries find application in strechable electronics, controllable stiffness devices, sensors and actuators. $^{14,15}$ Nevertheless, the purely mechanical actuation of wrinkling/creasing in film/substrate systems ${ }^{16,17}$ does not allow for an efficient active control of such surface patterns.

Bilayer surface patterning and stiffness control has also been obtained fairly recently by differential

a LMS, C.N.R.S., École Polytechnique, Université Paris-Saclay, 91128 Palaiseau, France.

*Email: konstantinos.danas@polytechnique.edu

$\dagger$ Electronic Supplementary Information (ESI) available: Electronic supplementary information (ESI) available: ESI includes a pdf (giving additional details on the experimental procedure and the finite element modeling) and a video (showing the wrinkling instability under combined magneto mechanical loading). See DOI: 10.1039/c7sm00996h] growth/swelling $6,18,19$ as well as by electroactive wrinkling and creasing ${ }^{20-22}$. Such externally applied stimuli can induce a biaxial compressive stress state within the film, which leads to buckling patterns (2D wrinkles or creases). Albeit effective as a method of pattern control, differential swelling usually requires large times of exposure to the absorbed water/solvents, thus leading to slow on/off pattern switching. On the other hand, electro(-mechanical) actuation is extremely fast but complex experimental setups have been used to obtain wrinkling patterns, e.g., dielectrics in conductive solutions and fairly high voltages. In both cases, the proposed setups are not straightforward for use in haptic devices but see recent advances in ${ }^{23}$.

In particular, challenged by the reduction of the actuation fields and the fast on/off control of surface patterns with the view of a realistic haptic device in mind, we propose in this work the use of novel polymeric-based soft materials that exhibit magnetoelastic coupling ${ }^{24-29}$ and can become unstable at small, practically attainable, magnetic fields ${ }^{30-34}$. Specifically, magnetorheological elastomers (MRE), i.e., ferromagnetic particle-impregnated elastomers, exhibit relatively large deformations (in the order of $10-20 \%$ ), while their response is extremely fast (in the order of milliseconds) upon application of a magnetic field. Under appropriate material design, internal reorganization of the fields occurs as the magnetization vectors of the particles tend to align with the externally applied field. As a result, magnetically triggered instabilities emerge in a reversible and repeatable fashion, when the particle microstructure and macroscopic geometry is unfavorably set with respect to the applied magnetic field ${ }^{34,35}$. 
From a novel perspective and by taking advantage of the magnetomechanical coupling, such material systems are potentially capable of operating near and beyond "marginally stable" regimes ${ }^{35,36}$. However, albeit potentially applicable in sensors, actuators and haptic devices ${ }^{37,38}$, a non-linear magnetoelastic system for active control of surface roughness has not been devised yet. Moreover, most efforts have been concentrated in maximizing the magnetostrictive coupling in MREs to increase the resulting deformations, with little success so far.

In this work, following a less common approach, we exploit experimentally and numerically the stability and postbifurcation response of a novel MRE film/substrate material system by proper control of magnetomechanically induced surface wrinkling. Precisely, we combine cooperative instabilities in such a way that surface wrinkles can be triggered by successively smaller magnetic fields as a result of increasing mechanical pre-compression and vice versa. To explore the impact of the magnetomechanical loading on the critical loads and bifurcation modes, we present a stability phase diagram of the system in the parameters space of mechanical precompression and applied magnetic field. The task is carried out first experimentally by introducing an innovative fabrication for perfectly-bonded MRE film/substrate polymer blocks and then numerically by employing a user-defined finite element full-field approach.

\section{Magnetomechanical Experiments, Morpho- logical Patterns and Bifurcation Amplitudes}

The experimental system consists of an elastomeric cube of $40 \mathrm{~mm}$ side length, in which an isotropic MRE film of thickness $H_{f}=0.8 \mathrm{~mm}$ and shear modulus $G_{f}$ rests on a nonmagnetic substrate of thickness $H_{S}=39.2 \mathrm{~mm}$ and shear modulus $G_{s}$. The two layers are made of elastomers of the same family and are cured simultaneously to prevent film delamination. The system is subjected to in-plane uniaxial compression perpendicular to the film thickness denoted by the stretch measure $\lambda_{1} \equiv \lambda_{0}=1+\varepsilon_{0}$ such that $0<\lambda_{0}<1$ (or $-1<\varepsilon_{0}<0$ ). The magnetic field $b_{0}$ is then linearly increased beyond the bifurcation point to obtain well-formed wrinkling.

\subsection{Fabrication and material properties}

The substrate is fabricated from silicone elastomer Ecoflex 00-10, mixed in a 1:1 weight ratio (polymer:hardener). The composite MRE film is similarly fabricated from silicone elastomer Ecoflex 00-50, uniformly mixed with $5 \mu \mathrm{m}$-diameter carbonyl iron particles at volume fraction $c=20 \%$. The film compound is poured into a metallic $40 \times 40 \times 0.8\left(\mathrm{~mm}^{3}\right)$ mold base. Scraping the top surface of the MRE material leads its excess quantity to flow out of the mold base, thus creating a

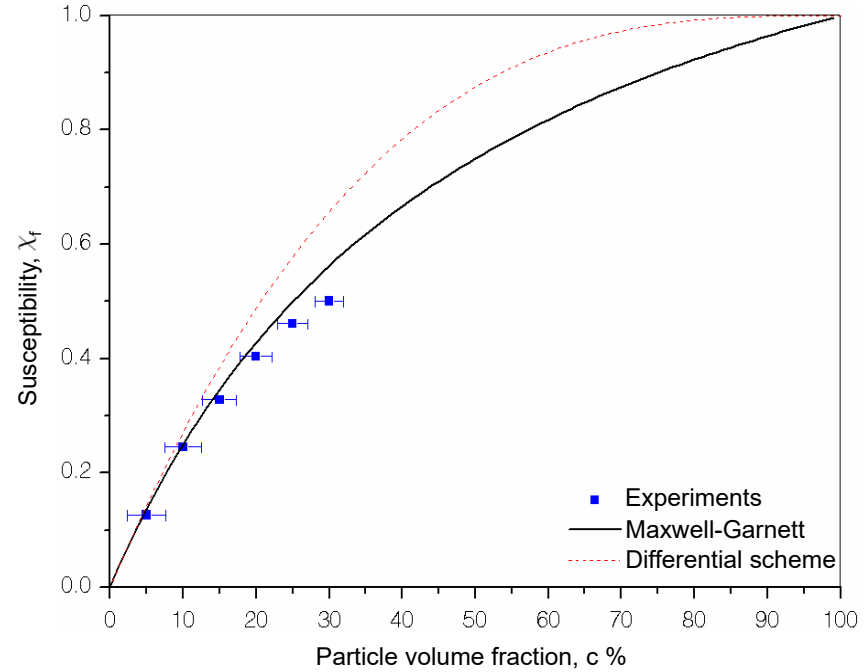

Fig. 1 Experimental measurements and analytical estimates of the MRE film relative susceptibility $\chi_{f}=\left(\mu_{f}-\mu_{0}\right) / \mu_{f}$, with $\mu_{f}$ and $\mu_{0}$ denoting the magnetic permittivity of the film and the air, respectively. The analytical homogenization Maxwell-Garnett and differential scheme curves are also included for comparison.

flat film surface. To build the whole $40 \times 40 \times 40\left(\mathrm{~mm}^{3}\right)$ system, four metallic walls are mounted onto the aforementioned base to form a taller mold. The substrate is subsequently poured on the top of the film. Heating resistors, attached to each of the walls, are connected to a temperature on/off PID regulation system delivering current, so that the temperature measured by a thermocouple (inserted in one of the walls) matches the programmed heating schedule. By virtue of this system, the film/substrate block is subjected to a $60 \mathrm{~min}$ curing process at $70^{\circ} \mathrm{C}$ with a $5^{\circ} \mathrm{C} / \mathrm{min}$ rate of temperature rise from ambient. The polymerization is carried out in the absence of a magnetic field, thus leading to an isotropic MRE film. Observations of a cross-section of the bilayer material with a VHX-5000 series Keyence Digital Microscope confirm a nearly constant film thickness $(0.8 \pm 0.04 \mathrm{~mm})$ and the complete absence of film delamination.

The MRE permeability $\mu_{f}$ is measured with a Bartington MS2G sensor and is used to obtain the relative film susceptibility $\chi_{f}$ from the relation $\chi_{f}=\left(\mu_{f}-\mu_{0}\right) / \mu_{f}$, with $\mu_{0}=$ $4 \pi 10^{-7} \mathrm{~N} A^{-2}$ the susceptibility of the air. The effect of the iron-particle volume fraction $c$ on the overall magnetic susceptibility (or permittivity) of the MRE composite film is shown in Fig.1. For completeness, we present two homogenization estimates, obtained by considering air permittivity $\mu_{0}$ for the silicone matrix and infinite permittivity for the iron particles (that is a good assumption since results do not change significantly if one considers a permittivity 100 times or more than that of the air). The Maxwell-Garnett estimate is given by 
$\mu_{f} / \mu_{0}=(1+2 c) /(1-c)$, whereas the differential scheme is given by $\mu_{f} / \mu_{0}=(1-c)^{-3}$. It is straightforward from Fig.1 that the Maxwell-Garnett is in better agreement with experiments. By setting $c=0.2$ (i.e., 20\%), one finds $\chi_{f}=0.4$, which is the value used in the subsequent numerical simulations.

The saturation magnetization of the MRE material is independent of the mechanical properties and the microstructure and is obtained by the simple rule of mixtures ${ }^{39}$. By using earlier experimental results in similar materials ${ }^{29}$, we use for the carbonyl iron a saturation magnetization in the order of $\mu_{0} m_{s}^{i r o n} \sim 2.5 \mathrm{~T}$, which gives $\mu_{0} m_{s}^{f}=0.5 \mathrm{~T}$ for the MRE film if mixed at $c=20 \%$ with a non-magnetic polymeric matrix.

To obtain the shear moduli of the substrate and the MRE film, we have used two bifurcation curves obtained experimentally at pre-compression $\lambda_{0}=0.8$ and 0.85 (see Fig 5a for a detailed discussion). Given that both the MRE film and the substrate are nearly incompressible, Lamé compressibility moduli that are $\sim 100$ times larger than the shear moduli of each phase have also been considered. From the fitting of the experimental bifurcation curves, we obtain the shear moduli for the substrate $G_{s}=3 \mathrm{KPa}$ and for the MRE film $G_{f}=$ $10 \mathrm{KPa}$, leading to a substrate-to-film ratio of $G_{s} / G_{f}=0.3$. These values are shown in Section 3 to be sufficient to probe the rest of the experimental curves accurately. It should be noted at this point that we have also carried out an independent uniaxial tension test on the substrate material in order to validate its response in the nonlinear range. The test was done at a very low strain rate $\left(\dot{\varepsilon}=5 \times 10^{-6} \mathrm{~s}^{-1}\right)$ using a standard dogbone sample and gave a Neo-Hookean response up to $10 \%$ nominal axial strain. Also, regarding the material response of the MRE, we note that it is not straightforward to have direct access to its properties when deposited as a film. Neither is straightforward to assume that the response of the MRE as a film will behave exactly as it would in a dogbone type specimen due to fabrication uncertainties and possible particle clustering during the deposition of the film*. Therefore, we will also assume for simplicity a Neo-Hookean response for the MRE film and we show in Section 3 that it is sufficient to probe the experimental bifurcation curves.

For later use, it is noted that for the present nearly incompressible Neo-Hookean solids with substrate-to-film shear moduli ratio $G_{s} / G_{f}=0.3$ the theoretical mechanical critical bifurcation stretch is $\lambda_{0}^{c} \simeq 0.76$.

* For the shear modulus of the substrate and the film, we also have to note that the curing process of a $40 \times 40 \times 40\left(\mathrm{~mm}^{3}\right)$ block leads to different polymerization time/process than in standard thin-section dogbone samples. Moreover, the film/substrate system is pre-compressed in a fixed setup and thus is in a fully relaxed state, while a tension experiment has intrinsic rate effects. Therefore, the uniaxial tension test in the substrate material is used as a guide and not as an independent fitting procedure.

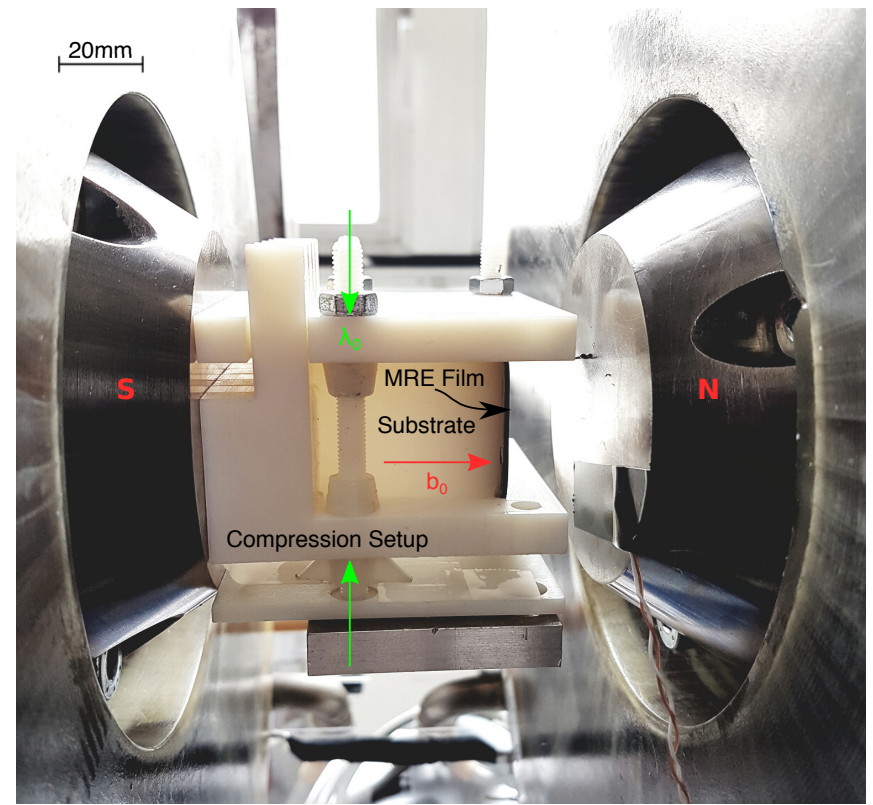

Fig. 2 Experimental setup depicting the magnetic poles, the 3D-printed compression device and the MRE film/substrate specimen.

\subsection{Experimental setup}

In the magnetomechanical bifurcation experimental setup, the compression device is 3D-printed using a non-magnetic polymeric material. It consists of three orthogonal walls forming a $U$ shape (see Fig.2). A non-magnetic screw-driven system is used to control the distance between the two parallel walls, so as to impose the prescribed compressive stretch ratio $\lambda_{0}$. The entire system is installed within the $82 \mathrm{~mm}$-air gap separating the two $90 \mathrm{~mm}$-diameter poles of a two-coil electromagnet (see Fig. 2). An external sensor is used to measure the amplitude of the applied magnetic field.

We capture the morphological side patterns of the film using a digital camera system with $12 \mu \mathrm{m} /$ pixel resolution and we trace the out-of-plane surface displacements as a function of the magnetic field, after image processing and analysis (see Supplementary Information). The images are recorded during testing at a rate of 17 frames per $0.1 \mathrm{~T}$.

\subsection{Experimental measurements}

The morphological response of the MRE film/substrate system is summarized in Fig.3a-p. Therein, the block, initially precompressed with $\lambda_{0}=0.95,0.85,0.8$ and 0.75 (Fig.3a,e,i,m), bifurcates as a result of the applied magnetic field $b_{0}$. The post-buckling evolution of the modes versus the applied magnetic field is also considered for the aforementioned $\lambda_{0}$ (Figs.3c-d,g-h,j-1,n-p). This magnetomechanical morpholog- 

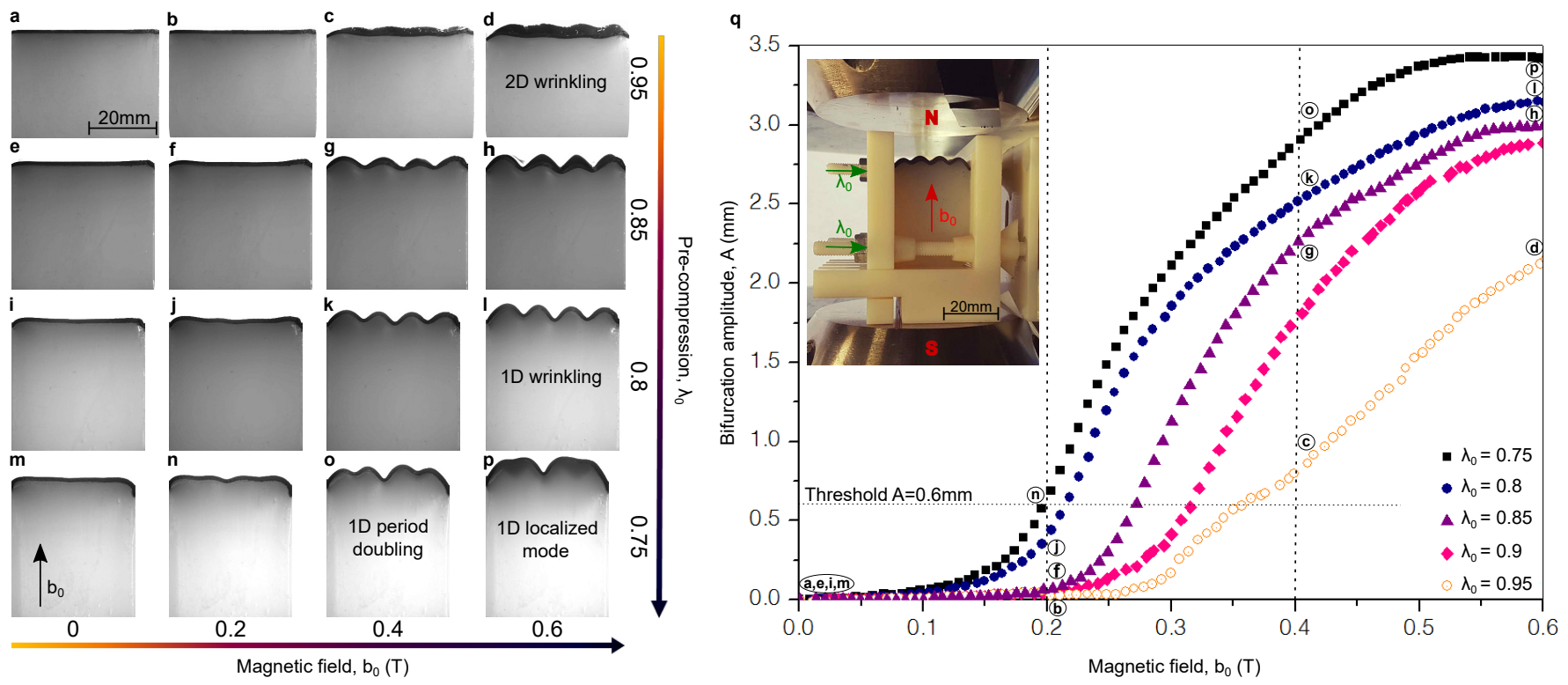

Fig. 3 Experimental influence of the magnetomechanical coupling on bifurcation and post-bifurcation. a-p, Optical images of the morphological pattern evolution versus the magnetic field $b_{0}$, under different pre-compressions $\lambda_{0}$. Scale bar, 20mm. Bifurcation patterns and critical loads depend on coupling: (a-d) For small pre-compressions, two-dimensional (2D) wrinkling patterns (see inset of Fig.5b blue region) are mainly controlled by the magnetic field, e.g., $\lambda_{0}=0.95, b_{0}^{c}<0.4 \mathrm{~T}$; (e-l) For moderate pre-strains, one-dimensional (1D) wrinkling pattern of stable wavenumber $\omega(=4)$ is obtained. The single-period wrinkling is the most preferable buckling mode in the range of pre-compressions tested. As pre-compression increases, wrinkling is triggered at smaller magnetic fields, e.g., $\lambda_{0}=0.85, b_{0}^{c}>0.2 \mathrm{~T}$ and $\lambda_{0}=0.8, b_{0}^{c} \approx 0.2 \mathrm{~T} ;(\mathbf{m}-\mathbf{p})$ For high pre-compressions, combination of wrinkling and period-doubling is observed while the critical magnetic fields do not decrease further, e.g., $\lambda_{0}=0.75, b_{0}^{c}<0.2 \mathrm{~T}$. q, Measurements of the bifurcation amplitude $A$ as a function of the magnetic field $b_{0}$, for different pre-compressions $\lambda_{0}$. $A$ in mm denotes the average distance between peaks and valleys of the two central wrinkles. For small pre-compressions (e.g., $\lambda_{0}=0.95$ ), the amplitude measurements should be analyzed with caution since the camera has no access to the internal part of the film surface, where higher wrinkling amplitude is developed (see Fig.5b-inset of blue regime).

ical map provides experimental evidence of different surface patterns formed with the same material system at different precompressions and magnetic fields. The morphology of the critical modes evolves more drastically with increasing precompression, while it remains almost unchanged with increasing magnetic field once in the post-bifurcation regime. The magnetoelastic properties of such a structure allow for bifurcation modes of sinusoidal type (wrinkles) within a large range of pre-compressions, even when $\lambda_{0}$ is significantly higher (i.e., compressive strain is lower) than the theoretical mechanical bifurcation point for a substrate-to-film shear moduli ratio of $G_{s} / G_{f}=0.3$, denoted as $\lambda_{0}^{c} \simeq 0.76$ (Fig.3a-l). For small precompressions, e.g., $\lambda_{0}=0.95$ (Fig.3a-d), the wrinkling patterns are complex and two-dimensional (2D) as a consequence of the high in-plane symmetries of the cubic block (i.e., almost square top surface of the film at small pre-compression) (but see also ${ }^{13,40}$ ).

By further pre-compressing, e.g., $\lambda_{0}=0.85,0.8$ (Fig.3e-1 and Supplementary Movie S1), the cubic symmetry breaks, leading to a one-dimensional (1D) sinusoidal wrinkling pattern of single period and stable wavenumber $\omega(=4)$. This mode governs the post-bifurcated response for a wide range of $\lambda_{0} \in[0.75,0.9]$, illustrating the similarity and cooperation of the magnetic and mechanical bifurcation modes. This similarity results from the substrate-to-film infinite magnetic susceptibility contrast $\left(\chi_{s} / \chi_{f}=0\right)$ and moderate mechanical moduli ratio $\left(G_{s} / G_{f}=0.3\right)$, respectively.

Further pre-compression beyond $\lambda_{0}=0.75$ (Fig.3m-p), causes single-period wrinkling to evolve into period-doubling. In this latter regime, the film/substrate block is under the influence of finite strains and significant friction on its lateral faces that are in contact with the mechanical compression device. This friction is manifested by the presence of a non-negligible curvature at the extremal sides of the film (see Figs.3i,m). This tends to inhibit the full formation of mechanical wrinkling of the film, even though we are at the theoretical critical mechanical load for wrinkling $\left(\lambda_{0}^{c} \simeq 0.76\right)$. Instead, it promotes a mechanical localized mode that resembles closely a crease mode but without self-contact of the free surface. By contrast, the application of the magnetic field gives rise to pure wrinkles. This suggests that at such large pre-compression the mechanical and magnetic bifurcation modes become dif- 
ferent and thus their cooperative nature is lost. As a consequence, the critical magnetic field saturates as a function of pre-compression, whereby its quantitative interpretation can already be observed in Fig. $3 q$ but is also discussed in detail in the context of Fig.5b. Further increase of the magnetic field leads to large out-of-plane deformations that relaxes the neighboring wrinkles and promotes a single localized pre-crease mode, as shown in Fig.3p. All the above-discussed morphological patterns reversibly vanish with slight hysteresis after magnetic loading removal (Supplementary Movie S1).

In the morphological map, one can qualitatively observe a monotonic tendency of the critical magnetic field to decrease with increasing pre-compression (and vice versa) (Fig.3c,f$\mathrm{g}, \mathrm{j}, \mathrm{n})$. To further explore quantitatively the influence of magnetomechanical coupling over the critical loads, we measure the evolution of the out-of-plane deflection of the filmreferred in this work as bifurcation amplitude $A$-as a function of the applied magnetic field $b_{0}$ (Supplementary Information). In Fig.3q, we summarize the supercritical bifurcation amplitude curves for different pre-compressions, which plainly depict the decreasing trend of the critical magnetic field as a function of pre-compression. The transition from the principal solution $(A=0)$ to the supercritical bifurcated branch is smooth and increases gradually with the magnetic field, indicating the presence of unavoidable geometrical and material imperfections deriving from the fabrication process. For later use, we define the bifurcation transition points $\left(\lambda_{0}, b_{0}^{c}\right)$ by setting a threshold over the amplitude $A(=0.6 \mathrm{~mm})$, as seen in Fig.3q. The threshold here is chosen so that clear wrinkles have been formed and is in the same range of the film thickness $H_{f}$.

\section{Numerical Modeling and Analysis}

To gain better understanding of the experiment, we investigate numerically the boundary value problem of the MRE film/substrate block by means of a user-element routine implemented in the general purpose finite element code FEAP ${ }^{41}$. The reason for using a full numerical analysis of the problem at hand will become more clear in the following. Nevertheless, it is perhaps worth mentioning at this point that in the post-bifurcation regime the stress, strain and magnetic fields become highly heterogeneous inside and outside of the specimen ${ }^{42,43}$ since the magnetic field is applied relatively far from the specimen and it is of Eulerian nature ${ }^{39}$. Those magnetic boundary conditions are very different than those in an electroactive experiment when the electrodes are directly attached on the film surface. In the later case, the stresses (Maxwell and mechanical ones) outside the film are zero while the applied electric field is of Lagrangian nature and follows the material deformation. In addition to this particularity, which is present in most magnetoelastic experiments, in the present ex- periment, one can easily observe in Figs.3j,m corresponding to large pre-compressions $\left(\lambda_{0} \leq 0.8\right)$ a non-negligible curvature at the lateral face of the film/substrate block which is in contact with the experimental setup. This curvature, which is a result of frictional effects, affects (as we will see below) the pre- and post-bifurcation response and needs to be taken into account in the present analysis. For all those reasons, we resort to a full numerical analysis of the boundary value problem at hand.

\subsection{Geometry and Mathematical Formulation}

Specifically, we consider a two-dimensional plane-strain (i.e., the stretch $\lambda_{3}=1$ in direction $X_{3}$ ) block surrounded by air, as shown in Fig.4. The dimensions of the solid phases (i.e., MRE film and passive substrate) are set equal to the experimental ones. The size of the surrounding air region ensures magnetic field uniformity far from the specimen in the free air space (Fig.4a). Due to the finite strains, a significant part of the air needs to be deformed via a penalty-function method, in order to avoid severe mesh distortion near the film/air interfaces (Supplementary Information). By numerically mimicking the experimental procedure, we first apply a pre-compressive stretch $\lambda_{0}$ in direction $X_{1}$ on the right side of the film/substrate block and then we linearly increase the magnetic field $b_{0}$ in direction $X_{2}$ (Fig.4b).

The experimentally observed friction at the lateral faces between the block and the walls of the compression device is only modeled approximately by prescribing tangential forces $f_{2}$ along direction $X_{2}$ (see Fig.4). These mechanical shear forces are increased linearly with the applied normal compressive stretch $\lambda_{0}$. The application of these shear forces results in a curvature of the lateral face of the MRE film similar to the experimental observations in Figs.3j,m. This point is rather technical and is further detailed in the Supplementary Information.

Continuity of the displacements across the film/substrate and film/air interfaces is automatically satisfied by use of nodal elements, while the tangential component of the magnetic vector field $\mathbf{b}$ is allowed to jump at these interfaces. In the present boundary value problem, the non-uniformity of the magnetic field at the corners of the film geometry, as well as the applied friction, are unavoidable sources of imperfections, leading to the numerical bifurcation curves in Fig.5a without any need for user-defined geometrical imperfections.

In order to deal with the finite strains and large magnetic fields, we work in a Lagrangian setting and define the deformation gradient $\mathbf{F}=\operatorname{Grad} \mathbf{x}=\mathbf{I}+$ Gradu and its determinant $J=\operatorname{det} \mathbf{F}>0$, with $\mathbf{x}=\mathbf{X}+\mathbf{u}(\mathbf{X})$ denoting the position vector of a material point in the deformed configuration, $\mathbf{X}$ the position vector of the same point in the reference configuration and $\mathbf{u}$ the displacement vector. This implies that the refer- 


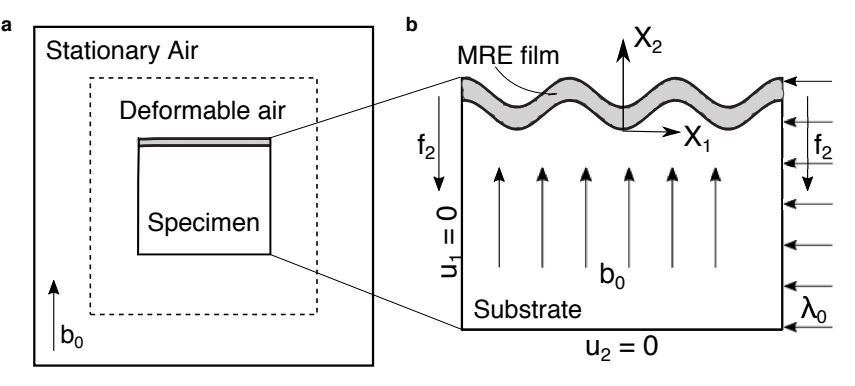

Fig. 4 Schematics of the numerical boundary value problem. a, MRE film adhering to a soft substrate under plane strain conditions, surrounded by deformable and stationary air (Supplementary Information). b, The block is initially subjected to uniaxial pre-compression $\lambda_{0}$ and tangential forces $f_{2}$ to mimic friction between the specimen and the compression device. Subsequently, the magnetic field $b_{0}$ is uniformly applied at the exterior boundary of the air far from the block and perpendicular to the film, i.e., along direction $\mathrm{X}_{2}$

ence density of the solid $\rho_{0}$ is related to the current density $\rho$ by $\rho_{0}=\rho J$. We also define the relation between the current (Eulerian) magnetic field $\mathbf{b}$ and reference (Lagrangian) one $\mathbf{B}$ via $\mathbf{b}=J^{-1} \mathbf{F B}$. Using the minimum energy formulation of Dorfman and Ogden ${ }^{25,28}$ and neglecting the purely mechanical body (gravity) forces, the potential energy $\mathscr{P}$ of the system may be expressed in terms of the displacement field $\mathbf{u}$ and the magnetic vector potential $\mathbf{A}(\mathbf{B}=\mathrm{Curl} \mathbf{A})$ such that

$$
\begin{aligned}
\mathscr{P}(\mathbf{u}, \mathbf{A}) & =\int_{\Omega_{i}} \rho_{0}^{i} \Phi_{i}(\mathbf{F}, \mathbf{B}) \mathrm{d} \Omega+\int_{\mathfrak{R}^{3}} \frac{1}{2 \mu_{0} J}\|\mathbf{F} \cdot \mathbf{B}\|^{2} \mathrm{~d} \Omega \\
& -\int_{\partial \Omega_{i}^{t}} \mathbf{T} \cdot \mathbf{u} d S
\end{aligned}
$$

for $i=$ film $(f)$, substrate $(s)$. Here, $\|$.$\| denotes the standard$ Euclidean norm, $\rho_{0}^{i}$ is the reference density and $\Phi_{i}$ denotes the Helmholtz free energy of the solids in the reference volume $\Omega_{i}$. The second term in (1) serves to describe the background magnetic (Maxwell) energy in the entire space $\mathfrak{R}^{3}$ and thus it accounts for all three phases, i.e., film, substrate and air. $\mathbf{T}$ is the mechanical surface traction vector applied at the traction part of the boundary of the reference volume $\partial \Omega_{i}^{t}$. The stationarity of the first variation of the potential energy (1) with respect to the independent variables $\mathbf{u}$ and $\mathbf{A}$ reads

$$
\delta \mathscr{P}=\mathscr{P}_{, \mathbf{u}} \delta \mathbf{u}+\mathscr{P}_{, \mathbf{A}} \delta \mathbf{A}=0
$$

(with ',' denoting partial derivation). Use of the vector potential $\mathbf{A}$, such that $\mathbf{B}=$ CurlA, together with the stationarity conditions in (2) yield the strong form of the Maxwell and equilibrium field equations ${ }^{25}$

$$
\operatorname{Curl} \mathbf{H}=0, \quad \operatorname{Div} \mathbf{B}=0, \quad \operatorname{Div} \mathbf{S}=\mathbf{0}, \quad \in \Omega_{i},
$$

where the operators Curl and Div are identified with respect to the reference configuration. The first Piola-Kirchhoff stress $\mathbf{S}$ and the Lagrangian magnetic h-field $\mathbf{H}$ are then given by

$$
\begin{aligned}
& \mathbf{S}=\rho_{0}^{i} \frac{\partial \Phi_{i}}{\partial \mathbf{F}}+\frac{1}{\mu_{0} J} \mathbf{B}(\mathbf{F} \cdot \mathbf{B})-\frac{1}{2 \mu_{0} J}\|\mathbf{F} \cdot \mathbf{B}\|^{2} \mathbf{F}^{-T}, \\
& \mathbf{H}=\rho_{0}^{i} \frac{\partial \Phi_{i}}{\partial \mathbf{B}}+\frac{1}{\mu_{0} J} \mathbf{F}^{T} \cdot(\mathbf{F} \cdot \mathbf{B}), \quad \text { in } \Omega_{i},
\end{aligned}
$$

For the definition of the free energy, it suffices in the present study (see discussion in Section 2.1 and Supplementary information) to propose a simple combination of a Neo-Hookean elastic energy and a Langevin (without hysteresis) magnetic energy such that

$$
\begin{aligned}
& \rho_{0}^{i} \Phi_{i}(\mathbf{F}, \mathbf{B})=\frac{G_{i}}{2}\left(\mathbf{F}^{T}: \mathbf{F}-3-2 \ln J\right)+\frac{G_{i}^{\prime}}{2}(J-1)^{2} \\
& +\frac{J \mu_{0}\left(m_{i}^{s}\right)^{2}}{3 \chi_{i}}\left[\ln \left(\frac{3 \chi_{i}\|\mathbf{b}\|}{\mu_{0} m_{i}^{s}}\right)-\ln \left(\sinh \left(\frac{3 \chi_{i}\|\mathbf{b}\|}{\mu_{0} m_{i}^{s}}\right)\right)\right],
\end{aligned}
$$

where it is noted that $\|\mathbf{b}\|=\sqrt{\mathbf{b} \cdot \mathbf{b}}=J^{-1} \sqrt{(\mathbf{F} \cdot \mathbf{B}) \cdot(\mathbf{F} \cdot \mathbf{B})}$. In equation (5), $G_{i}$ is the shear modulus, $G_{i}^{\prime}\left(=100 G_{i}\right)$ the second Lamé compressibility constant and $\mu_{0}$ the permeability of vacuum. Langevin's function expresses to a good approximation the magnetoelastic response of the MRE film composite as a function of its magnetic material properties, i.e., the saturation magnetization $\mu_{0} m_{s}^{f}$ and the susceptibility $\chi_{f}$ (see Section 2.1). As discussed in Section 2.1, the use of a simple Neo-Hookean mechanical response for the MRE material constitutes a fair approximation, which accurately probes the experimental post-bifurcation amplitudes for the entire range of the loading states considered in this study. In turn, the substrate is non-magnetic implying a magnetic susceptibility $\chi_{s}=0$, which in turn leads to the vanishing of the last term in (5) (for $i=$ substrate) and thus is described by a simple purely mechanical Neo-Hookean model. The nearly incompressible response of the film/substrate system imply an out-of-plane deformation $\lambda_{2} \simeq 1 / \lambda_{0}$, in accord with the experimental measurements. This is satisfied via a large Lamé compressibility constant in the above-described constitutive laws of the solids.

The numerical calculations are carried out by use of standard four-node quadrilateral bilinear isoparametric elements, with 3 degrees of freedom per node: the displacements $\mathbf{u}=$ $\left\{u_{1}\left(X_{1}, X_{2}\right), u_{2}\left(X_{1}, X_{2}\right), 0\right\}$ and the magnetic vector potential $\mathbf{A}=\left\{0,0, A_{3}\left(X_{1}, X_{2}\right)\right\}$, where $\left(X_{1}, X_{2}\right)$ denote the global reference coordinates. The nonlinear solutions of the field equations were obtained incrementally with the use of a standard Newton-Raphson scheme. A more detailed description of the numerical algorithms and meshes is presented in the Supplementary Information. 


\subsection{Experiments versus Numerical Simulations}

The experimental (post-)bifurcation amplitudes are then successfully probed with the full-field finite element calculations. For clarity, we show in Fig.5a such comparisons for three values of pre-compression. It is important to note that the amplitude curves corresponding to a pre-compression $\lambda_{0}=0.8,0.85$ have been used to identify the shear moduli of the MRE film $\left(G_{f}=10 \mathrm{KPa}\right)$ and the substrate $\left(G_{s}=3 \mathrm{KPa}\right)$, thus leading to a substrate-to-film shear moduli ratio $G_{s} / G_{f}=0.3$.

The rest of the numerical estimates are then found to be in good agreement with the experimental measurements for the aforementioned loading parameters and are capable to reproduce extremely well the decrease of the critical magnetic field with increasing pre-compression. The agreement between the numerical simulations and the experiments is also very good in terms of the obtained patterns as shown in the inset of Fig.5a. The numerical results predict exactly four wrinkles along the film length. This fair comparison allows us to use the FEM results in the following section to investigate in detail the resulting magnetic fields in the post-bifurcation regime.

Using next the experimental and numerical curves together with the threshold value $A(=0.6 \mathrm{~mm})$, we propose a comprehensive two-field stability phase diagram in Fig.5b. The stability map shows that pattern switching can be achieved by proper control and cooperation of the magnetic and mechanical fields. In addition, the reduction of the critical magnetic field $b_{0}^{c}$ with increasing pre-compression is experimentally quantified and numerically probed. For instance, when the block is under significant pre-compression (e.g., $\lambda_{0}=0.8$ ), the critical magnetic field $b_{0}^{c}(=0.2 \mathrm{~T})$ reduces to half of that for small mechanical pre-compression (e.g., $\lambda_{0}=0.98, b_{0}^{c}=$ $0.38 \mathrm{~T})$. Furthermore, based on pattern distinction, this diagram can be qualitatively divided into three representative domains: 2D wrinkling (blue), 1D wrinkling (white) and a 1D period-doubling (see Fig.3o) regime (orange) that is followed by a pure mechanical localized (pre-crease) mode at $\lambda_{0}=0.7$ as shown in the inset picture.

At smaller pre-compressions (blue region), we observe 2D wrinkling as a result of high biaxial in-plane symmetry of the film/substrate block and no particular bias to plane-strain modes. In this case, the numerical and experimental results exhibit a slight deviation. This could be easily explained by noting on one hand that in the numerical results we assume plane-strain conditions and on the other hand that our camerasetup has only access to the side wrinkling amplitude. Instead, in this case of low pre-compression, the maximum amplitude occurs in the middle of the specimen (see inset Fig.5b in blue regime). In turn, at moderate-to-high pre-compressions (especially for $\lambda_{0}<0.85$ ), the experimental and numerical bifurcation points $\left(\lambda_{0}, b_{0}^{c}\right)$ are in excellent agreement, as a result of the uniquely prescribed plane-strain conditions. This result vali- dates qualitatively the simplified bifurcation analysis carried out by Danas and Triantafyllidis ${ }^{35}$, albeit in different MREs.

Interestingly, the critical $b_{0}^{c}$ field decreases linearly with the pre-compression $\lambda_{0}$ up to the theoretical mechanical bifurcation point $\lambda_{0}^{c} \simeq 0.76$. Beyond that point, an asymptotic response is observed with no further reduction of $b_{0}^{c}$ (orange regime in Fig.5b). This asymptotic saturation of the critical magnetic field at large pre-compression is a direct consequence of friction and curvature effects at the lateral faces of the film as discussed in the context of Fig.3m-p. As a result of this curvature effects, the mechanical (localized pre-crease mode in Fig.3n) and the magnetic (wrinkling Fig.3o) bifurcation modes become different and thus non-cooperating. As a consequence of this non-cooperation, no further decrease of the critical magnetic field $b_{0}^{c}$ is obtained with increase of precompression. One could probably improve upon this with a thinner film. Then, the number of wrinkles would increase (wavenumber is an inverse function of film thickness) and boundary effects would become less important. The influence of friction upon the observed bifurcation mode is, however, by itself an interesting result that requires further investigation and is briefly discussed in the Supplementary Information for the present geometry.

To conclude Fig.5b, the critical field reduction is clearly demonstrated for the first time and it is a direct consequence of the magnetomechanical coupling and the proper cooperation of the resulting mechanical and magnetic instabilities. The qualitative explanation behind it is the following: the elastic energy stored by pre-compression $\lambda_{0}$ brings the system near but not exactly to a mechanically critically stable state, which aids in turn to trigger a cooperative magnetic instability at a lower magnetic field $b_{0}^{c}$. The low magnetic fields at play (e.g. one can easily touch the film surface during the experiment and feel the roughness at a $\mathrm{mm}$ scale) and the simplicity of the proposed MRE film/substrate geometry makes the present system a potential candidate for an actual magnetomechanical device that allows for dynamic active control of surface patterns at relatively small magnetic fields. It is worth also noting that the critical magnetic field can be further reduced by more unstable microstructures such as particle-chain ones ${ }^{35}$. Such work is underway and will be presented in the future.

\subsection{Full-field numerical analysis}

To understand better the magnetomechanical bifurcation, we show representative contour plots from the full-field simulations in Fig.6a-f, omitting the surrounding air. The MRE film/substrate system is at a post-bifurcated state at $b_{0}=0.4 \mathrm{~T}$ exhibiting periodic wrinkling for $\lambda_{0}=0.8$ (white regime in Fig.5b). The spatial distribution of the Green-Lagrange strains $\mathbf{E}$ is shown in Fig.6a,b, whereas the corresponding magnetic field $\mathbf{b}$ and the magnetization $\mathbf{m}$ are depicted in Fig.6c,d 

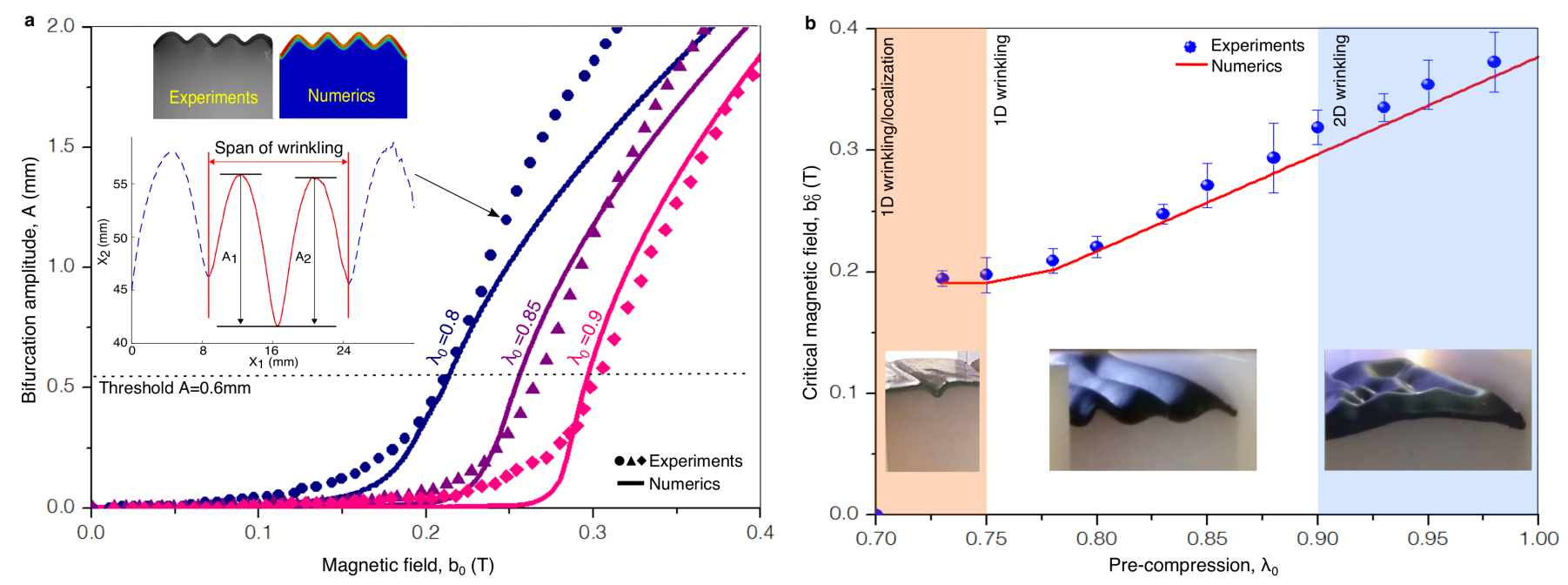

Fig. 5 Identification of bifurcation in the two-field parameter space: mechanical pre-compression $\lambda_{0}$ and magnetic field $b_{0}$. A decreasing trend of the critical magnetic field $b_{0}^{c}$ as a function of the applied pre-stretch $\lambda_{0}$ is observed in: $\mathbf{a}$, Supercritical bifurcation curves for $\lambda_{0}=0.8,0.85,0.9$. Continuous lines correspond to numerical predictions, while symbols correspond to experimental data reported in Fig.3q. The insets present, at $\lambda_{0}=0.8$ and $b_{0}=0.25 \mathrm{~T}$, comparisons between experimental and numerical profiles, as well as the experimental film profile used to obtain the amplitude curves. b, A two-field stability phase diagram of morphological patterns. Experimental and numerical $\left(\lambda_{0}, b_{0}^{c}\right)$ critical points for 2D wrinkling (blue), 1D wrinkling (white) and wrinkling followed by period-doubling patterns (orange) are defined from the bifurcation curves (represented in Figs.3q, 5a and S5a), by considering a threshold of macroscopically observed wrinkling amplitude $A=0.6 \mathrm{~mm}$. Standard deviation of measurements among 3 specimens is included in experimental data points.
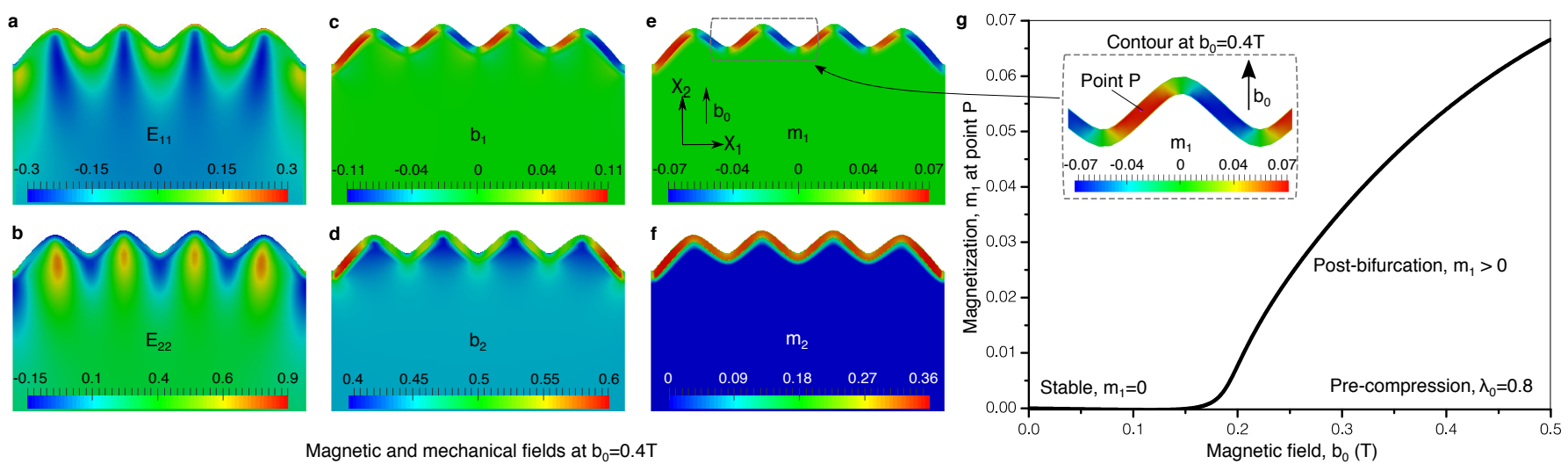

Fig. 6 Numerical post-bifurcation results for the MRE film/substrate system at pre-compression $\lambda_{0}=0.8$ and $b=0.4 \mathrm{~T}$. Simulation parameters: $H_{f}=0.8 \mathrm{~mm}, H_{s}=39.2 \mathrm{~mm}, G_{f}=10 \mathrm{KPa}, G_{s}=3 \mathrm{KPa}, \chi_{f}=0.4, \mu_{0} m_{s}^{f}=0.5 \mathrm{~T}$. a-f, Deformed configuration and contour plots of the spatial distribution of the Green-Lagrange strains, magnetic fields and magnetization along $X_{1}$ and $X_{2}$ directions. Part of the substrate is not shown for clarity. g, Supercritical bifurcation curve of magnetization $m_{1}$ as a function of the applied magnetic field $b_{0}$, at a fixed point $P$ within the film.

and Fig.6e,f, respectively. The strain fields are classical for such problems, i.e., bending deformation state within the film, which slowly dies out and becomes more uniform within the substrate with increasing distance from the film/substrate interface.

In turn, the magnetic fields display an interesting distribution pattern. The components $b_{2}$ and $m_{2}$, which are in the di- rection of the applied magnetic field $b_{0}$ are more pronounced near the corners, while they remain positive in the entire film and substrate. By contrast, the perpendicular to the applied field $b_{0}$ components $b_{1}$ and $m_{1}$ exhibit symmetrical alternating patterns ranging from negative to positive values. While these fields are locally heterogeneous, on average, they can be thought of as magnetic phase domains with interchanging 
magnetization direction. This further enhances the wrinkling amplitude and, consequently, the overall deformation of the film/substrate system upon increase of the applied magnetic field $b_{0}$, until magnetic saturation is reached.

It should be noted at this point that the magnetic fields $b_{1}$ and $b_{2}$ (and hence the corresponding Maxwell stresses) are highly heterogeneous and non-zero outside the film. That is of course a direct consequence of the Eulerian applied magnetic field far from the specimen ${ }^{39,42,43}$. This makes the analytical treatment of the problem very difficult and any approximations introduced to deal with the post-bifurcation response should be done with extreme caution.

For completeness, in Fig.6g, we show the magnetization component $m_{1}$ at a representative point $\mathrm{P}$ located within the MRE film with positive values of $m_{1}$ (see inset of that figure). At this point $P$, the MRE displays a supercritical pitchfork bifurcation response as a function of the applied magnetic field $b_{0}$. This magnetization bifurcation curve is similar to the bifurcation amplitude curves shown in Fig.5, but currently can only be accessed via full field numerical simulations.

Finally, it is worth mentioning that the present numerical model has only been used here to understand better the experimental response of the given MRE film/substrate system. The fact, however, that it has also proven to be quantitatively accurate allows us to use it as such in future studies to explore and design more optimized MRE film/substrate systems in an extended geometrical and material parameter space. Such work is underway and will be presented elsewhere.

\section{Concluding Remarks}

In this study, we have investigated the active control of surface roughness by exploiting the post-bifurcation response of a critically stable MRE film bonded to a non-linear elastic and highly compliant substrate. The key idea is to mechanically bring the structure near (but not at) a marginally stable state and then destabilize it with relatively small magnetic fields. As shown experimentally and numerically, this decrease of critical magnetic fields works only when the bifurcation modes and critical loads are cooperative. In simple words, one needs the bifurcation modes triggered by the first field, say the mechanical one, to be the same (or very similar) to the modes induced by the second field, i.e., the magnetic field. Then, one can achieve a noteworthy reduction of the critical magnetic field with increasing pre-compression. In any other case where the instability patterns are not similar, the two fields act somehow independently and even though they do not bring further decrease of the critical magnetic fields for bifurcation, they do lead to an otherwise impressive superposition of different modes (such as wrinkles triggered by the magnetic field and pre-creased localized modes from the mechanical one), as shown in Fig.3o.
The experimental results were probed successfully with the aid of full-field finite element simulations at finite strains and large magnetic fields. For this, we have used a simple nonlinear magnetoelastic Helmholtz free energy with magnetization saturation for the MRE film, a classical non-linear elastic constitutive law for the substrate and direct simulation of the surrounding air. This allowed to reach a quantitatively good agreement between the experimental measurements and the numerical results, while having access to the complete contours of the magnetic and mechanical fields. These fields are heterogeneous inside both solids and across the film in the post-bifurcation regime and in principle cannot be resolved analytically. The numerical simulations allow, however, for the detailed study of those fields and better understanding of the post-bifurcation response. The post-bifurcation response has been shown to be supercritical both mechanically and magnetically, thus allowing to magnetically load/unload the material system in a cyclic and reversible manner, switching on and off the morphological patterns. The numerical model, finally, can be utilized for a systematic study and optimization of such material systems.

It should be mentioned that the film/substrate system exhibits a relatively small hysteretic response upon unloading 44 but this effect has not been studied in this work. The sources for such hysteresis are potentially the viscoelastic nature of the polymers as well as the magnetic hysteresis of iron. The latter, however, is found in earlier studies ${ }^{29}$ to be rather small and thus in the present problem the main source of hysteresis is believed to be the viscoelasticity of the polymers. To distinguish between the two mechanisms in a robust manner, one has to analyze the problem with a fully dissipative magnetoelastic theory. Such work is in progress.

In closing, the combination of the experiments and numerical simulations presented in this study are encouraging and could potentially open the way for a realistic instabilitytriggered polymer-based magnetorheological device for the active control of surface patterns at small magnetic fields. Moreover, the response is scalable and thus, the present system could also be built at the micron scale with more advanced fabrication techniques. Finally, the present idea of using two (or more) fields to control instabilities in the post-bifurcation regime is more general and can be used in any coupled/active material system if properly designed ${ }^{22,39}$.

\section{Acknowledgements}

This work was supported by the European Research Council (ERC) under the European Unions Horizon 2020 research and innovation program (grant agreement No 636903 - MAGNETO). 


\section{References}

1 J.-Y. Sun, S. Xia, M.-W. Moon, K. H. Oh and K.-S. Kim, Proc. Royal Soc. A, 2012, 468, 932-953.

2 J. W. Hutchinson, Phil. Trans. Royal Soc. A, 2013, 371, 0422.

3 L. Jin and Z. Suo, J. Mech. Phys. Solids, 2015, 74, 68-79.

4 P. Kim, M. Abkarian and H. A. Stone, Nat Mater, 2011, 10, 952-957.

5 Q. Wang and X. Zhao, J. App. Mech., 2013, 81, 051004.

6 Y. Zhao, X. Han, G. Li, C. Lu, Y. Cao, X.-Q. Feng and H. Gao, J. Mech. Phys. Solids, 2015, 83, 129-145.

7 L. Jin, A. Auguste, R. C. Hayward and Z. Suo, J. App. Mech., 2015, 82, 061008.

8 S. Cai, D. Breid, A. Crosby, Z. Suo and J. Hutchinson, J. Mech. Phys. Solids, 2011, 59, 1094 - 1114.

9 S. Cai, D. Chen, Z. Suo and R. C. Hayward, Soft Matter, 2012, 5, 13011304.

10 A. Auguste, L. Jin, Z. Suo and R. C. Hayward, Extr. Mech. Lett., 2017, 11, 30-36.

11 L. Pocivavsek, R. Dellsy, A. Kern, S. Johnson, B. Lin, K. Y. C. Lee and E. Cerda, Science, 2008, 320, 912-916.

12 J. Zang, X. Zhao, Y. Cao and J. W. Hutchinson, J. Mech. Phys. Solids, 2012, 60, $1265-1279$.

13 N. Stoop, R. Lagrange, D. Terwagne, P. M. Reis and J. Dunkel, Nat Mater, 2015, 14, 337-342.

14 K. Bhattacharya and R. James, J. Mech. Phys. Solids, 1999, 47, 531 576.

15 S. Lacour, J. Jones, Z. Suo and S. Wagner, IEEE Electron Device Lett., 2004, 25, 179-181.

16 N. Bowden, S. Brittain, A. G. Evans, J. W. Hutchinson and G. M. Whitesides, Nature, 1998, 393, 146-149.

17 W. T. S. Huck, N. Bowden, P. Onck, T. Pardoen, J. W. Hutchinson and G. M. Whitesides, Langmuir, 2000, 16, 3497-3501.

18 V. Trujillo, J. Kim and R. C. Hayward, Soft Matter, 2008, 4, 564-569.

19 E. P. Chan, E. J. Smith, R. C. Hayward and A. J. Crosby, Advanced Materials, 2008, 20, 711-716.

20 Q. Wang and X. Zhao, Physical Review E, 2013, 88, 042403.

21 S. Wang, M. Decker, D. L. Henann and S. A. Chester, Journal of the Mechanics and Physics of Solids, 2016, 95, 213 - 229.

22 H. Bense, M. Trejo, E. Reyssat, J. Bico and B. Roman, Soft Matter, 2017, 13, 2876-2885.

23 G. Frediani, D. Mazzei, D. De Rossi and F. Carpi, Frontiers Bioeng. Biotech., 2014, 2, 31.

24 M. R. Jolly, J. D. Carlson and B. C. Muñoz, Smart Mat. Struct., 1996, 5, 607.

25 A. Dorfmann and R. W. Ogden, Eur. J. Mech. A/Solids, 2003, 22, 497 507.

26 I. A. Brigadnov and A. Dorfmann, Int. J. Solids Struc., 2003, 40, $4659-$ 4674.

27 A. Dorfmann and R. W. Ogden, Quart. J. Mech. App. Math., 2004, 57, 599-622.

28 S. V. Kankanala and N. Triantafyllidis, J. Mech. Phys. Solids, 2004, 52, $2869-2908$.

29 K. Danas, S. Kankanala and N. Triantafyllidis, J. Mech. Phys. Solids, 2012, 60, $120-138$.

30 S. Kankanala and N. Triantafyllidis, J. Mech. Phys. Solids, 2008, 56, 1147 -1169 .

31 C. R. Tipton, E. Han and T. Mullin, Soft Matter, 2012, 8, 6880-6883.

32 S. Rudykh and K. Bertoldi, J. Mech. Phys. Solids, 2013, 61, $949-967$.

33 F. Gerbal, Y. Wang, F. Lyonnet, J.-C. Bacri, T. Hocquet and M. Devaud, Proc. Nat. Acad. Sci., 2015, 112, 7135-7140.

34 S. Huang, G. Pessot, P. Cremer, R. Weeber, C. Holm, J. Nowak, S. Odenbach, A. M. Menzel and G. K. Auernhammer, Soft Matter, 2016, 12, 228
237.

35 K. Danas and N. Triantafyllidis, J. Mech. Phys. Solids, 2014, 69, 67 - 83.

36 Y. Grabovsky and L. Truskinovsky, J. Nonlin. Sci., 2013, 23, 891-969.

37 J. M. Ginder, M. E. Nichols, L. D. Elie and S. M. Clark, SPIE Proceedings 3985, Smart Structures and Materials 2000: Smart Structures and Integrated Systems, 2000, 3985, 418.

38 L. Lanotte, G. Ausanio, C. Hison, V. Iannotti and C. Luponio, Sens. Actuat. A: Phys., 2003, 106, 56-60.

39 K. Danas, J. Mech. Phys. Solids, 2017, 105, 25-53.

40 B. Audoly and A. Boudaoud, J. Mech. Phys. Solids, 2008, 56, $2401-$ 2421.

41 R. L. Taylor, FEAP - Finite Element Analysis Program, -, 2011.

42 M.-A. Keip and M. Rambausek, Int. J. Solids Struct., 2017, 121, 1 - 20.

43 V. Lefèvre, K. Danas and O. Lopez-Pamies, J. Mech. Phys. Solids, 2017, 107, 343-364.

44 R. H. J. Yoon, J. Kim, Soft Matter, 2010, 6, 5807. 